\title{
The volume dependence of spectral weights and the pentaquark state
}

\section{Constantia Alexandrou*}

Department of Physics, University of Cyprus, CY-1678 Nicosia, Cyprus

E-mail:alexandeucy.ac.cy

\section{Antonios Tsapalis}

University of Athens, Institute of Accelerating Systems and Applications, Athens, Greece

E-mail: tsapalisecc.uoa.gr

Before studying the pentaquark system we examine the spectral weights of the two lowest scattering states in the two-pion system in the isospin $\mathrm{I}=2$ channel on lattices of size $16^{3} \times 32,24^{3} \times 32$ and $32^{3} \times 64$ at $\beta=6.0$ in the quenched theory. We find that the spectral weights scale with the volume for large time separations. Therefore very accurate data are necessary in order that the spectral weights determined on different volumes yield a ratio that is precise enough to distinguish a scattering state from a single particle state. The pentaquark system is studied on the same lattices and scaling of the spectral weights of the low lying state is investigated. The accuracy of the results obtained for the scaling of spectral weights do not allow us to exclude a pentaquark resonance.

XXIIIrd International Symposium on Lattice Field Theory

25-30 July 2005

Trinity College, Dublin, Ireland

\footnotetext{
*Speaker.
} 


\section{Introduction}

Several experiments performed during the past couple of years (see e.g. [1]) to search for the $\Theta^{+}$, an exotic baryon with an unusually narrow width, failed to confirm the signal that was originally reported in various low energy experiments [2]. This has cast doubts on the existence of this state and raised interesting questions regarding its production mechanism that add to the puzzle as to what its structure might be to explain its narrow width.

During the same time several quenched lattice calculations reached different conclusions as to the existence of a resonant five quark state. The main difficulty comes from the fact that the $\Theta^{+}$is not the lowest lying state but it is expected to be about $100 \mathrm{MeV}$ above the $\mathrm{KN}$ threshold. Identifying in an unambiguous way the resonance from the $\mathrm{KN}$ s-wave scattering state within lattice QCD, given the small energy gap, is a difficult task. One approach employed in a number of studies to distinguish them is to examine the volume dependence of local correlators [3]. Expanding the correlator computed on a lattice of spatial size $L$ in terms of eigenstates of the theory with the same quantum numbers as the interpolating field one obtains [ 4 ]:

$$
C_{L}(t)=\sum_{n=1}^{\infty} w_{L}^{n} e^{-E_{L}^{n} t}
$$

For a single particle state the spectral weights $w_{L}^{n}$ are approximately volume independent, whereas for a two particle scattering state well below resonance $w_{L}^{n} \sim 1 / L^{3}$. Besides the spectral weights one can examine the volume dependence of the energy spectrum. For two non-interacting particles $h_{1}$ and $h_{2}$ in the center of mass frame the energy is given by:

$$
E_{h_{1} h_{2}}^{n}=\sqrt{m_{h_{1}}^{2}+n\left(\frac{2 \pi}{L}\right)^{2}}+\sqrt{m_{h_{2}}^{2}+n\left(\frac{2 \pi}{L}\right)^{2}} \quad n=0,1,2, \ldots
$$

For $n>0$ the energy is volume dependent and it can be distinguished from the energy of a resonance, which is volume independent for large enough volumes. Before examining the pentaquark system we study the scaling of spectral weights for a simpler system of two pions in the isospin $I=2$ channel where we expect no low lying resonance. We use lattices of size $16^{3} \times 32,24^{3} \times 32$ and $32^{3} \times 64$ at $\beta=6.0$ with Dirichlet boundary conditions in the temporal direction. For a two particle scattering state the expected ratio of spectral weights for our three volumes is $w_{16} / w_{24}=3.4$, $w_{24} / w_{32}=2.4$ and $w_{16} / w_{32}=8$. All the results shown here are done taking $\kappa=0.153$ for the $\mathrm{u}$ and d- quark propagators corresponding to a pion mass of about $830 \mathrm{MeV}$.

\section{Two pion system}

We use local $I=2$ interpolating fields constructed by taking products of pion and rho fields:

$$
J_{1}(x)=J_{1}^{\pi}(x) J_{1}^{\pi}(x) \quad J_{2}(x)=J_{2}^{\pi}(x) J_{2}^{\pi}(x) \quad J_{3}(x)=J_{0}^{\rho}(x) J_{0}^{\rho}(x) \quad J_{4}(x)=\sum_{i=1}^{3} J_{i}^{\rho}(x) J_{i}^{\rho}(x)
$$

where $J_{1}^{\pi}(x)=\bar{d}(x) \gamma_{5} u(x), J_{2}^{\pi}(x)=\bar{d}(x) \gamma_{5} \gamma_{0} u(x), J_{0}^{\rho}(x)=\bar{d}(x) \gamma_{0} \sum_{i=1}^{3} \gamma_{i} u(x)$ and $J_{i}^{\rho}(x)=\bar{d}(x) \gamma_{i} u(x)$. Using the fields defined in Eq. (2.1) we construct a $4 \times 4$ correlation matrix $C_{j k}(t)=\sum_{x}\left\langle 0\left|J_{j}(x) J_{k}^{+}(0)\right| 0\right\rangle$. 

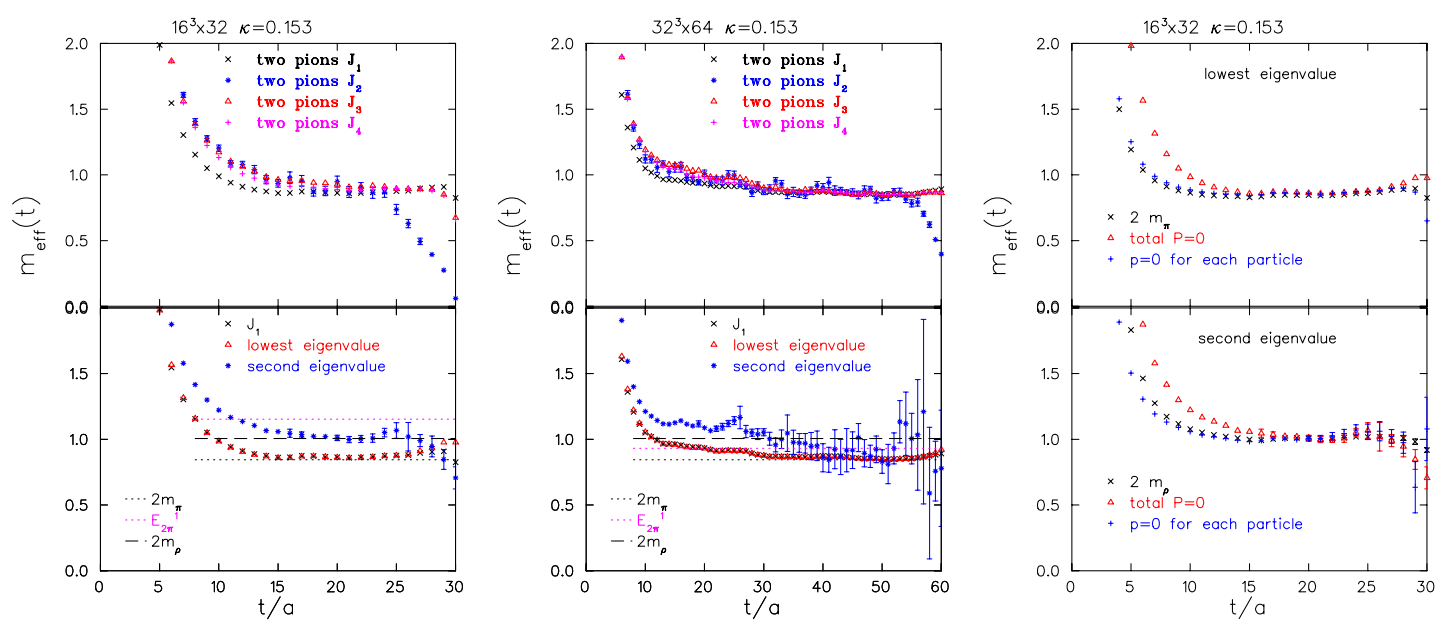

Figure 1: Upper left and middle graphs show effective masses using $J_{1}, J_{2}, J_{3}$ and $J_{4}$ on a lattice of size $16^{3} \times 32$ and $32^{3} \times 64$ respectively. Lower left and middle graphs show effective masses from $J_{1}$ and the two lowest eigenvalues. The graph on the right compares the effective mass for the two lowest eigenvalues using the unprojected correlation matrix and the projected correlation matrix of Eq. (2.2) for the lattice of size $16^{3} \times 32$.

In addition we project to a state in which each of the two particles carries zero relative momentum by evaluating the correlation matrix:

$$
C_{j_{s^{\prime}} k_{s}}(t)=\sum_{x, y}\left\langle 0\left|J_{j}^{s^{\prime}}(x) J_{j}^{s^{\prime}}(y) J_{k}^{s+}(0) J_{k}^{s+}(0)\right| 0\right\rangle \quad s, s^{\prime}=\pi, \rho
$$

Our variational methods of analysis are described in Ref. [5].

The effective masses for all interpolating fields are shown in Fig. 1 where one can see that they converge to the same plateau yielding the same value for the mass. This value is the same as that obtained from the lowest energy eigenvalue and very close to the mass of the s-wave twopion scattering state $E_{2 \pi}^{0}$, whereas the second eigenvalue yields $E_{2 \rho}^{0}$. The fact that the two lowest eigenstates correspond to s-wave scattering states can be explicitly demonstrated by analyzing the correlation matrix with projection to zero momentum for each particle as shown in the same figure for the lattice of size $16^{3} \times 32$. The masses extracted from the two lowest eigenvalues are shown as a function of the spatial size of the lattice in Fig. 2. The energy of the second eigenvalue is volume independent and can be clearly distinguished from $E_{2 \pi}^{1}$. Note that finite volume corrections due to particle interactions are too small to be seen on the scale of this graph. The fact that for the lattice of size $24^{3} \times 32$ the masses are slightly above $E_{2 \pi}^{0}$ and $E_{2 \rho}^{0}$ is due to the fact that the time extent of the lattice is too small to filter zero momentum pions and rho mesons. Also it is worth mentioning that the two-rho scattering state, although higher than $E_{2 \pi}^{1}$ on our largest lattice, is the dominant state in the intermediate time range and only for very large times the effective mass of the second lowest state becomes consistent with $E_{2 \pi}^{1}$.

In order to study the scaling of spectral weights $w_{L}$ we first look at the ratio of correlators computed on lattices of spatial size $L_{1}$ and $L_{2}$ multiplied by the corresponding effective masses:

$$
R_{L_{1}: L_{2}}=\frac{C_{L_{1}}(t) e^{m_{e f f, L_{1}}(t)}}{C_{L_{2}}(t) e^{m_{e f f, L_{2}}(t)}} .
$$




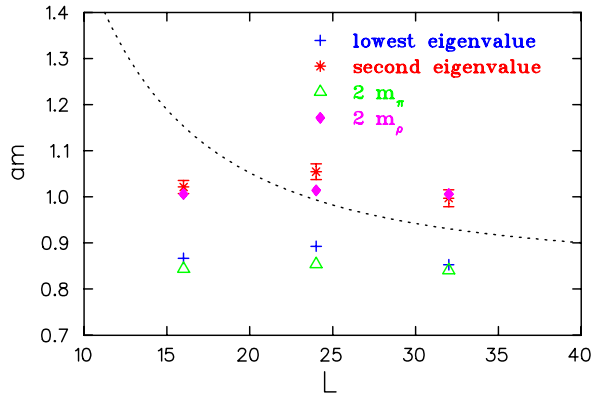

Figure 2: The mass extracted from the lowest and second lowest energy eigenvalue versus $\mathrm{L}$ in lattice units. The dotted line is $E_{2 \pi}^{1}$.

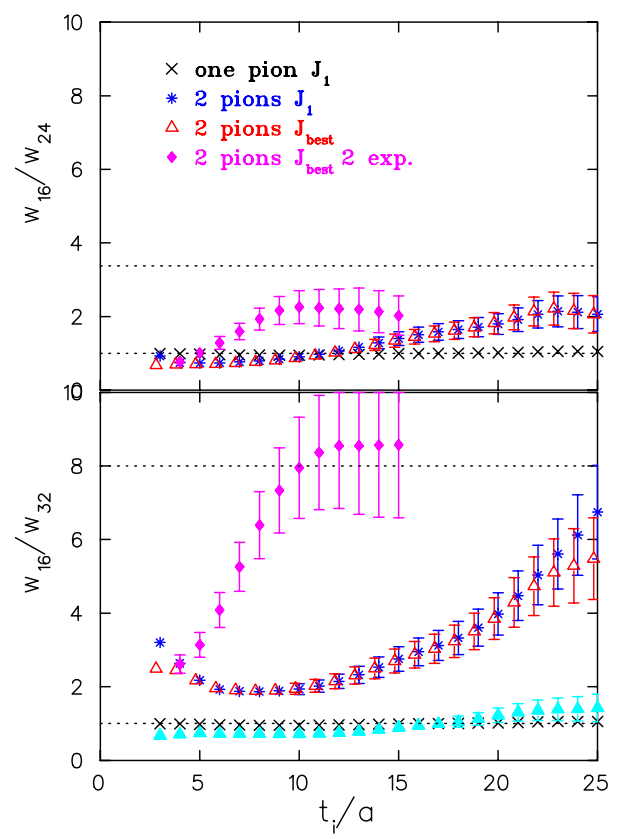

Figure 4: The ratio of spectral weights for the pion and the lowest state of the two-pion system. The dotted lines show the expected value of the ratio for a single particle and for a two particle scattering state. The results shown by the filled triangles in the lower graph are obtained taking the upper fit range for the large lattice to be 26 in lattice units.

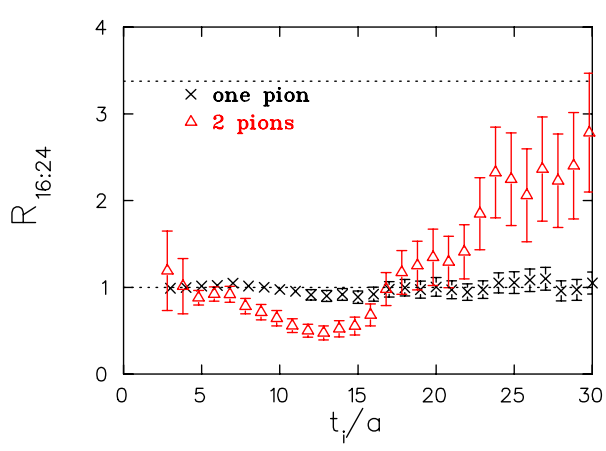

Figure 3: The ratio $R_{16: 24}(t)$ as a function of $t$.

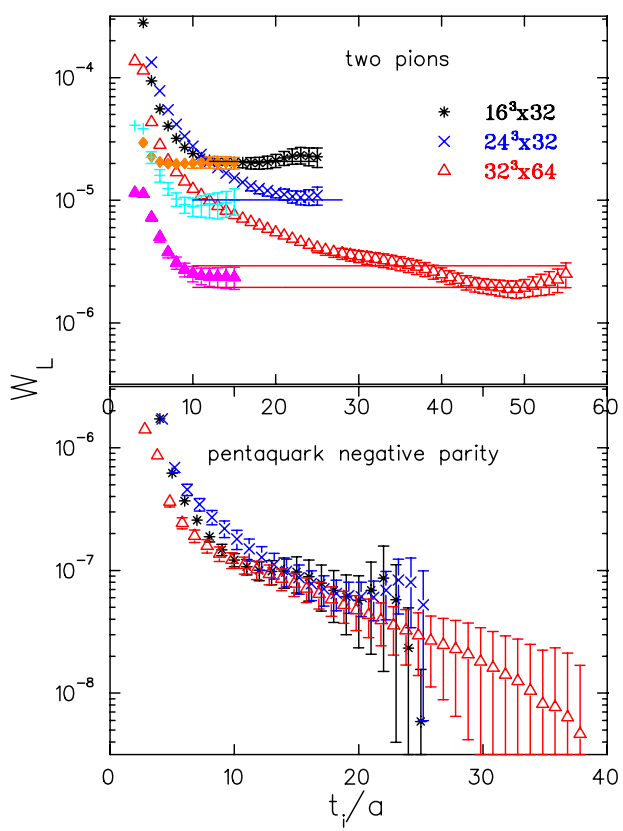

Figure 5: Spectral weights on our three lattices. Top for the two pion system and bottom for the pentaquark in the negative parity channel. In the pion system we show results from fits to a single exponential and a sum of two exponentials.

If a single state dominates then this ratio gives the ratio of spectral weights. We evaluate this ratio for the lattices of spatial extension 16 and 24. As can be seen in Fig. 3, for the one pion state this ratio is one as expected. For the two-pion state it increases but only approaches the expected value of 3.4 for $t / a>25$ when the ground state dominates. For the lattice of spatial size 32 this happens for $t / a>30$ and therefore this ratio is about one up to $t / a=30$, which is the maximum time separation that it can be constructed. A second option is to extract the spectral weights by fitting the correlators to one or a sum of two exponentials. This allows to take into account information from the full time extent of the lattice. We plot the ratio of spectral weights $w_{L_{1}} / w_{L_{2}}$ for our three lattices as a function of the lower time range $t_{i} / a$ used in the fit. The upper time range is fixed to 
26 for the lattices of time extent 32 and to 56 for the lattice of time extent 64 . As can be seen in Fig. 4 the ratio of weights deviates from one and approaches the expected ratio from below for large values of $t_{i} / a$. The individual spectral weights are shown in Fig. 5 and they are volume dependent. The values obtained by fitting the correlator to a sum of two exponentials are consistent with those obtained using a single exponential but converge at smaller values of $t_{i} / a$ on all lattices. However if instead of an upper fit range of 56 we take 26 for the $32^{3} \times 64$ lattice as we did for the other two then the ratio of spectral weights stays very close to one as can be seen in Fig. . The same analysis can be done for the second eigenstate but the errors are too large to reach a definite conclusion even for this simple system.

\section{Implications for the pentaquark system}

We use interpolating fields motivated by the diquark-diquark [6] and $\mathrm{KN}$ structure:

$$
J_{D D}^{I=0}=\varepsilon^{a b c}\left(u_{a}^{T} C \gamma_{5} d_{b}\right)\left[u_{c}^{T} C d_{e}-u_{e}^{T} C d_{c}\right] C \bar{s}_{e}^{T} \gamma_{5}, \quad J_{K N}^{I=0}=\varepsilon^{a b c}\left(u_{a}^{T} C \gamma_{5} d_{b}\right)\left[u_{c}\left(\bar{s} \gamma_{5} d\right)-d_{c}\left(\bar{s} \gamma_{5} u\right)\right] .
$$

We fix $\kappa_{s}=0.155$ for the strange quark. This choice gives $m_{K} / m_{N}=0.5$ and $m_{\phi} / m_{N}=1.04$ close

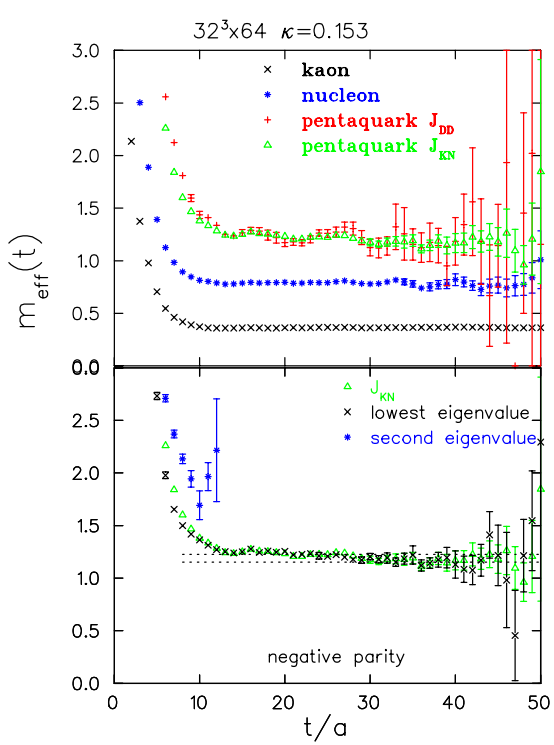

Figure 6: The effective mass for the pentaquark in the negative parity channel. Top: for $J_{D D}$ and $J_{K N}$. Bottom: for the two lowest eigenvalues. The dotted lines show the energies of the KN scattering states $E_{K N}^{0}$ and $E_{K N}^{1}$.

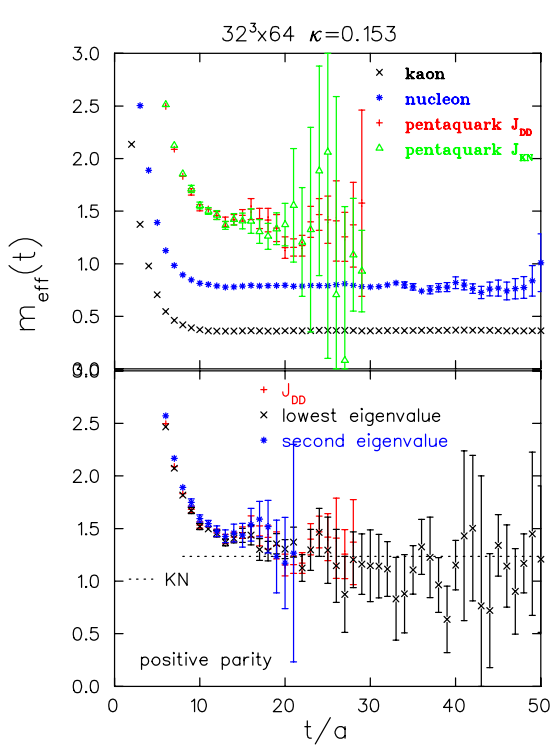

Figure 7: The same as Fig. 8 put for the positive parity channel. The dotted line is $E_{K N}^{1}$

to the experimental ratios. The effective masses for the negative and positive parity channels are shown in Figs. 6 and 7 for the $16^{3} \times 32$ and $32^{3} \times 64$ lattices. The plateau region is larger for the negative parity than for the positive parity channel with both interpolating fields yielding consistent results. For comparison we also show the effective mass for the nucleon and the kaon. We perform the same variational analysis as in the two pion system to extract the two lowest energy eigenvalues shown in Figs. 6and 7. What we find is that in the negative parity channel the second energy is very 
poorly determined whereas the lowest state is consistent with that obtained from either interpolating fields. Like in the two pion system the s-wave KN scattering state is obtained when $t / a>30$ for the large lattice. For the positive parity the two eigenvalues are very close together and cannot be accurately resolved. We find a mass splitting of roughly $100 \mathrm{MeV}$ at this quark mass. As in the two pion system only the ratio $R_{16: 24}$ is useful. For the $32^{3} \times 64$ lattice the ratio is not useful since one has to go beyond $t / a>30$ to see the scaling. Instead we extract the spectral weights by fitting the correlators to a single exponential using the same upper fit ranges as those used in the two-pion system. The results are shown in Fig. 5. In both the two-pion and pentaquark systems the values of the spectral weights on the two smaller lattices stabilize as we increase the lower fit range $t_{i} / a$ whereas on the large lattice convergence is slow. However within this variation the ratio of weights clearly deviates from unity. For the pentaquark system fitting to two exponentials is very noisy and only single exponential fits are performed. The values of the weights extracted on the three lattices show a very different behavior as compared to that observed in the two-pion system. Within the statistical errors they show no volume dependence for $10<t_{i} / a<20$ unlike in the two pion system where for the same time range a clear volume dependence is seen.

\section{Conclusions}

The mass correlation matrix constructed from operators that have the quantum numbers of the isospin $\mathrm{I}=2$ two pion system yields two eigenvalues that correspond to the two-pion and two-rho s-wave scattering states. Scaling of the spectral weights with the volume is verified but requires large time separations and accurate data. Even though for this system the statistical errors on the correlators for the first excited state are small the ratio of spectral weights for this state is still too noisy obscuring the volume dependence. Carrying the same analysis for the pentaquark system we find that, using the $\mathrm{KN}$ and the diquark-diquark interpolating fields, we obtain reliably only the lowest energy eigenvalue in the negative parity channel which, for large time separations, is consistent with $E_{K N}^{0}$. The spectral weights can only be accurately determined in the negative parity channel and for our three volumes they do not show strong volume dependence unlike what is observed in the pion system. Thus, to the accuracy with which the scaling of the spectral weights is determined, we cannot exclude a pentaquark resonance. In the positive parity channel we find that the two eigenvalues are very close in energy with a gap of about $100 \mathrm{MeV}$ at $\kappa=0.153$. However the mass of this state is too high to be identified as the $\Theta^{+}(1540)$.

\section{References}

[1] I. Abt et al., Phys. Rev. Lett. 93212003 (2003); J. Z. Bai et al., Phys. Rev. D 70:012004 (2004); S. Schael et al., Phys. Lett. B 599, 1 (2004) ; Yu. M. Antipov et al., Eur. Phys. J. A21, 455 (2004).

[2] T. Nakano, et al., Phys. Rev. Lett. 91, 012002 (2003); V. V. Barmin et al., Phys. Atom. Nucl. 66, 1715 (2003); S. Stepanyan et al., Phys. Rev. Lett. 91, 252001 (2003).

[3] N. Mathur et al., Phys. Rev. D 70, 074508 (2004).

[4] M. Lüscher, Nucl. Phys.B364, 237 (1991).

[5] C. Alexandrou and A. Tsapalis, hep-lat/0502016.

[6] S. Sasaki, Phys. Rev. Lett. 93152001 (2004). 